\title{
INTEGRATION OF A LOW-COST MULTISENSORY UAV SYSTEM FOR FOREST APPLICATION
}

\author{
Jianping $\mathrm{Li}^{1,{ }^{1}, \text { Bisheng Yang }}{ }^{1,}$, Yangzi Cong ${ }^{1}$, Senlei $\mathrm{Li}^{1}$, Yuanwen Yue ${ }^{2}$ \\ ${ }^{1}$ State Key Laboratory of Information Engineering in Survey, Mapping and Remote Sensing, Wuhan University, \\ No. 129, Luoyu Road, Wuhan, PR China - (lijianping, bshyang, yzcong, SenorLee)@whu.edu.cn \\ ${ }^{2}$ School of Resource and Environmental Science, Wuhan University \\ No. 129, Luoyu Road, Wuhan, PR China - yueyuanwen@whu.edu.cn
}

Commission II, WG II /3

KEY WORDS: Low-cost, Multi-sensor, UAV, Mobile mapping, Forest applications

\begin{abstract}
:
To integrate the multi-spectral imagery and laser scanning data for forest management, a low-cost multisensory UAV system, named Kylin Cloud, is introduced in this paper. The Kylin Cloud is composed several low-cost sensors (i.e., GNSS receiver, IMU, global shutter camera, multispectral camera, and laser scanner), providing the fusion of the imagery and laser scanning data for reliable forest inventory. Experiments were undertaken in a forest park in Wuhan. Results showed that the registration error of the multispectral Digital Orthophoto Map (DOM) and laser scanning data is about one pixel, demonstrating a high potential of the proposed low-cost system.
\end{abstract}

\section{INTRODUCTION}

Forest management relies on accurate spectral and geometrical characteristics of trees. Traditionally, the detailed information of trees is acquired through field inventory, which is labor-intensive, time-consuming (Dai et al., 2018; Wang et al., 2016; Yang et al., 2016). In these years, many efforts focused on the fusion of the imagery and laser scanning data and achieved effective improvement in the forest applications, i.e., species identification (Puttonen et al., 2010), habitat modeling (García et al., 2011), and land cover classification (Tooke et al., 2009). Although the integration of the spectral and geometrical data works effectively, accurate registration of two kinds of data from different platform is the prerequisite for data integration and a challenging task (Abayowa et al., 2015; Li et al., 2018). If one mobile mapping system could provide the fusion of the multispectral imagery and laser scanning data for reliable forest inventory, acquisition costs would be reduced and economic would be feasibility stimulated. Prospectively, forestry decisions are made based on the remote sensing surveys.

In this work, we are concerned with the integration of a low-cost multisensory UAV system for forest applications. A low-cost multisensory UAV system called Kylin Cloud is introduced. It is mainly composed of five sensors: a GNSS, an IMU, a global shutter camera, a laser scanner, and a multispectral camera. All of these sensors are pre-calibrated and time synchronized. Secondly, multispectral imagery and laser scanning data is georeferenced according to the direct georeferencing data. Then, accurate registration of the multispectral Digital Orthophoto Map (DOM) and laser scanning data is achieved.

The remainder of this paper is organized as follows. The proposed system and data integration are described in Section 2. In Section 3, the experimental studies are undertaken to evaluate the proposed method, after which conclusions are drawn at the end of this paper.

\section{METHODOLOGY}

\subsection{Configuration of the Low-cost Multisensory UAV System}

As illustrated in Fig.1, the UAV based multisensory mobile mapping system named Kylin Cloud is composed of five sensors a GNSS receiver, a low-cost IMU, a global shutter camera, a laser scanner, and a multispectral camera. The UAV is a DJI m600-pro with the maximum payload of $5 \mathrm{~kg}$ and 25 minutes flying time. The sensor specifications of the sensors are listed in Table 1 . The time synchronization of all the sensors is fulfilled electronically. For a more detailed description, we recommend readers see (Li et al., 2019).

The range error of the Velodyne Puck VLP-16 laser scanner is about $0.03 \mathrm{~m}$, whose accuracy is proved to be sufficient for the forest applications (Jaakkola et al., 2017). The Parrot sequoia multispectral camera is designed for analyzing plants' vitality by measuring the amount of light they absorb and reflect. The four separated bands of the camera, green (550 BP 40), red (660 BP 40), red edge (735 BP 10), and near infrared (790 BP 40). The Pointgrey color global shutter camera (Flea3) is set to the exposure rate of $5 \mathrm{~Hz}$. The Xsens Mti-300 IMU is configured with $200 \mathrm{~Hz}$ raw data (the linear acceleration and angular velocity). Xsens Mti-300 is a low-cost IMU (accelerometer inrun bias is $0.015 \mathrm{mg}$; gyroscope in-run bias is $10 / \mathrm{h}$ ). The accurate georeferencing data is calculated by integration the GNSS data, IMU data, and image sequence using the GNSS and IMU assisted structure from motion proposed in ( $\mathrm{Li}$ et al., 2019) to overcome the poor performance of the low-cost IMU.

\footnotetext{
* Corresponding author
} 


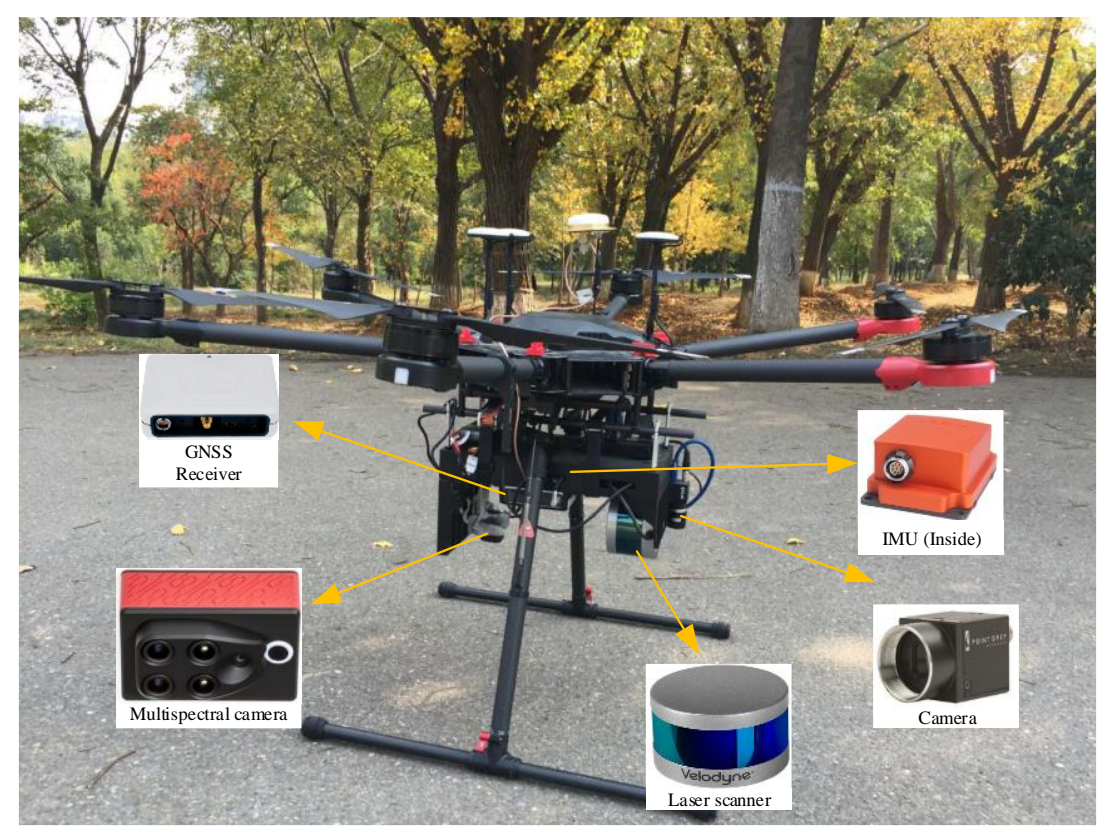

Fig.1 System description of Kylin Cloud. ( For a more detailed description, see (Dai et al., 2018; Li et al., 2019) )

\begin{tabular}{ccc}
\hline \hline Sensor & Manufacturer & Description \\
\hline GNSS receiver & P8 made by KQ GEO Technologies* & $\begin{array}{c}\text { Double frequency, supporting } \\
\text { BDS/GPS/GLONASS }\end{array}$ \\
IMU & Xsens MTI-300 & accelerometer in-run bias is $0.015 \mathrm{mg} ;$ \\
& gyroscope in-run bias is $10 / \mathrm{h}$ \\
Global shutter camera & Pointgrey Flea3 & $1280 \times 1024$ pixels with pixel size of $5.3 \mu \mathrm{m}$ \\
Laser scanner & Velodyne Puck VLP16 & 16 channels; 300,000 points per second; \\
Multispectral camera & Parrot sequoia & 4 bands: green, red, red edge, near infrared \\
\hline \hline
\end{tabular}

*KQ GEO Technologies is a Chinese company (http://www.kanq.com.cn/).

Table 1. Sensors specifications.

\subsection{Coordinate System Definition}

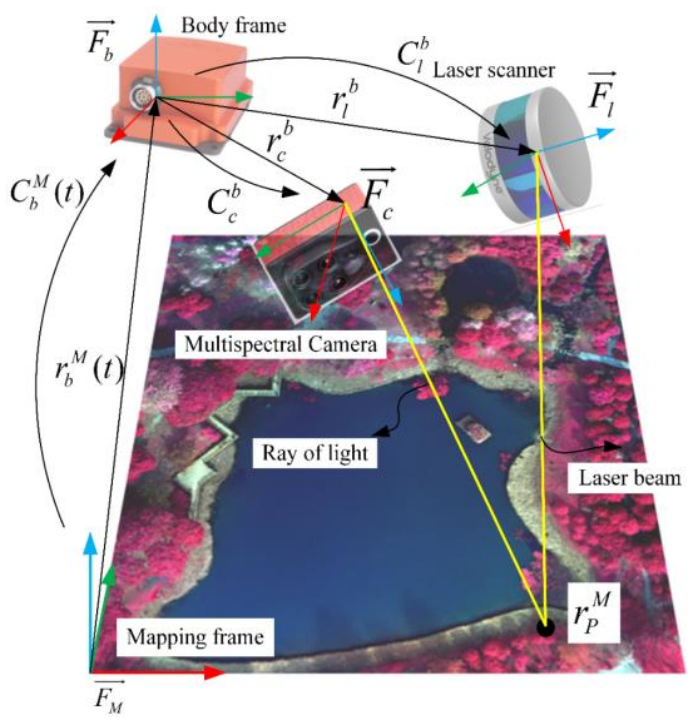

Fig.2 Coordinate definitions involved in the proposed multisensory UAV system.

The coordinate system of the proposed system is defined as follow: $\overrightarrow{F_{A}}$ represents a reference frame $A$. A point $\mathrm{P}$ in frame
$\overrightarrow{F_{A}}$ is written as $\boldsymbol{r}_{P}^{A}$. The rotation matrix $\boldsymbol{C}_{B}^{A}$ rotates a vector from $\overrightarrow{F_{B}}$ to $\overrightarrow{F_{A}}$. As shown in Fig.2, four coordinate system are used in the proposed multisensory UAV system: the mapping system $\left(\overrightarrow{F_{M}}\right)$, the body frame $\left(\overrightarrow{F_{b}}\right)$, the multispectral camera sensor frame $\left(\overrightarrow{F_{c}}\right)$, and the laser scanner frame $\left(\overrightarrow{F_{l}}\right)$. The transformation (rotation $\boldsymbol{C}_{b}^{M}(t)$ and translation $\boldsymbol{r}_{b}^{M}(t)$ ) between $\overrightarrow{F_{b}}$ and $\overrightarrow{F_{M}}$ at any certain time $t$ is derived from the estimated georeferencing data.

\subsection{Multisensory Data Registration}

Data preprocessing is to register the point clouds with multispectral Digital Orthophoto Map (DOM). By applying the Eq. (1), the point clouds are reconstructed in the mapping frame according to the measurements of laser scanner. Meanwhile, the Digital Surface Model (DSM) is calculated using the point clouds. Then the multispectral DOM is generated using the generated DSM and the raw multispectral images according to Eq. (2). The workflow is shown in Fig.3.

$$
\begin{aligned}
\boldsymbol{r}_{P}^{M} & =\boldsymbol{C}_{b}^{M}(t) \boldsymbol{C}_{l}^{b} \boldsymbol{r}_{P}^{l}+\boldsymbol{C}_{b}^{M}(t) \boldsymbol{r}_{l}^{b}+\boldsymbol{r}_{b}^{M}(t) \\
\boldsymbol{r}_{P}^{M} & =\boldsymbol{C}_{b}^{M}(t) \boldsymbol{C}_{c}^{b} \boldsymbol{r}_{P}^{c}+\boldsymbol{C}_{b}^{M}(t) \boldsymbol{r}_{c}^{b}+\boldsymbol{r}_{b}^{M}(t)
\end{aligned}
$$




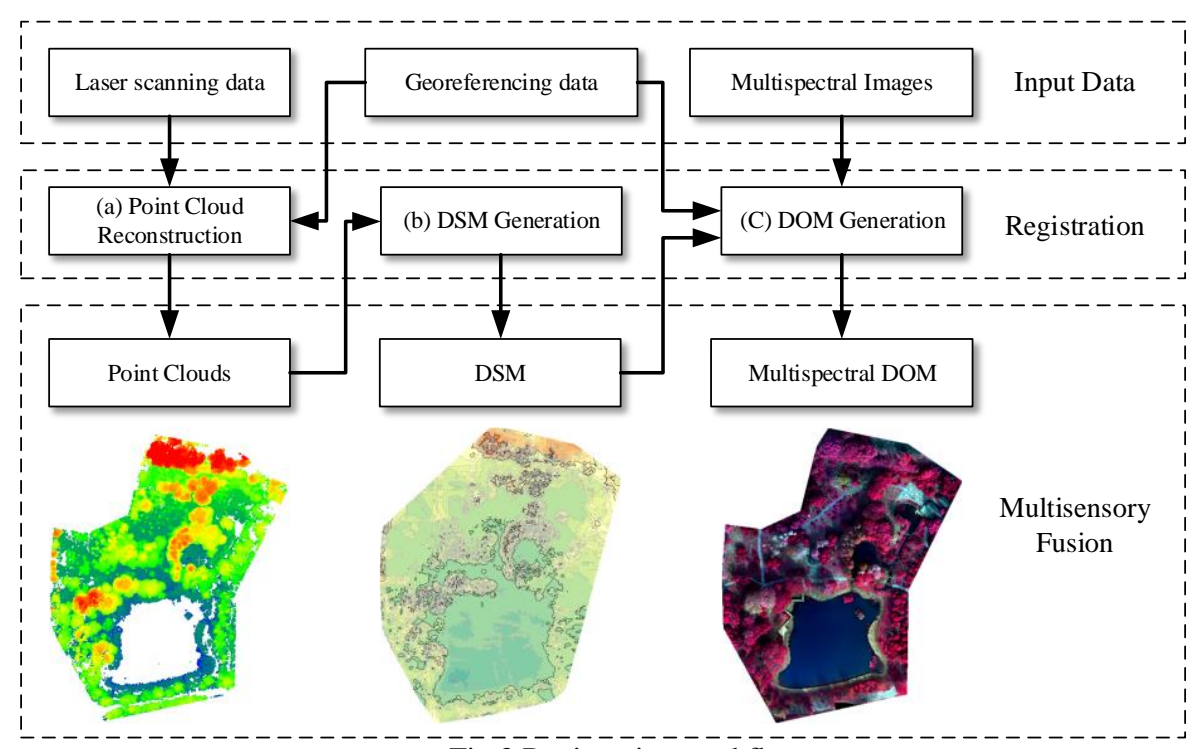

Fig.3 Registration workflow
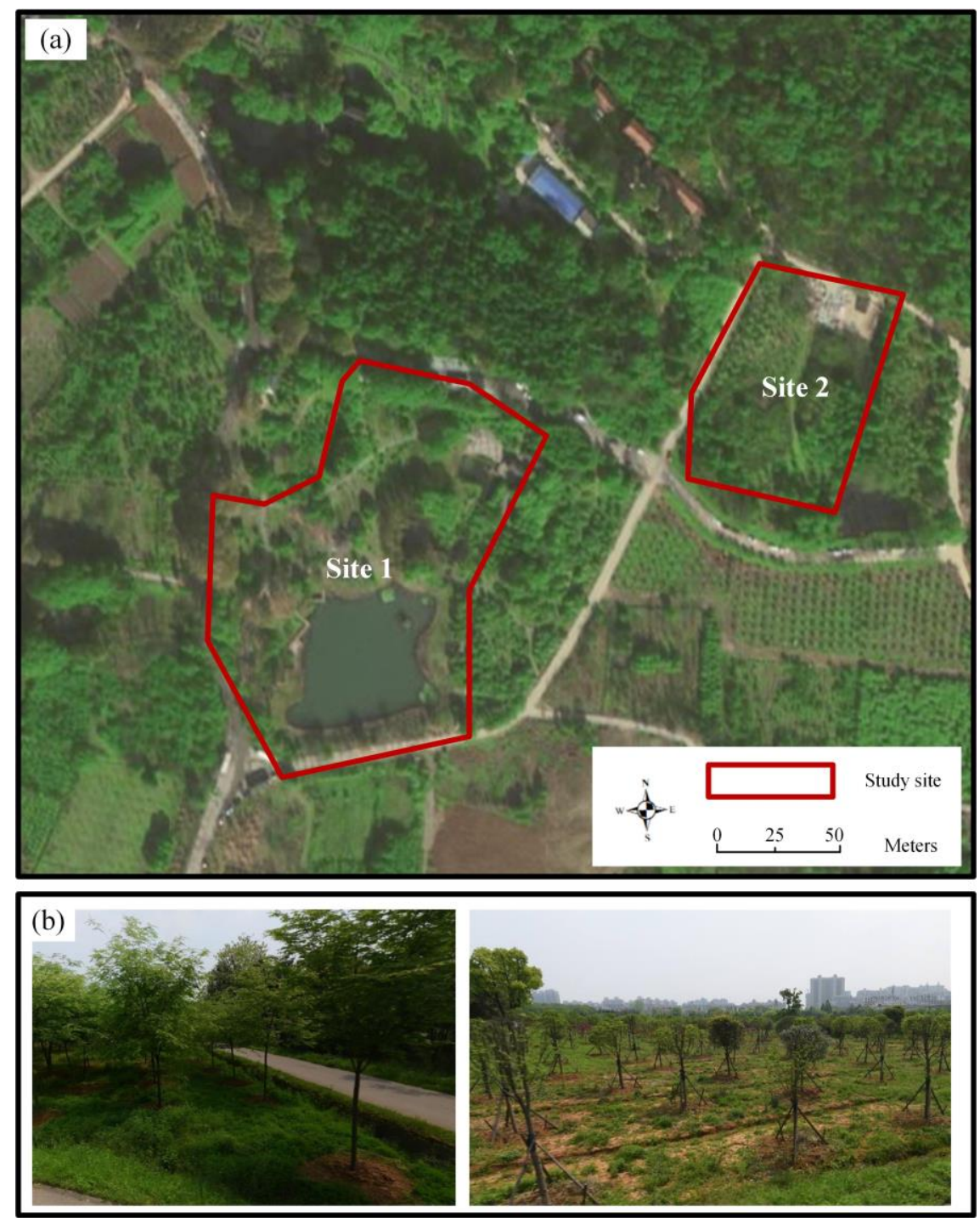

Fig.4 Locations of study site in Wuhan Maan Mountain forest park. (a) Location and orthophoto of the study sites. (b) Images of the trees in study sites. 


\section{EXPERIMENTS}

To validate the proposed low-cost multisensory UAV system, two sets of data were collected. Besides, the multisensory data is registered using the proposed method. The registration accuracy of the registration result is checked by corresponding points selected from laser scanning points and generated multispectral DOM. In this section, the detailed information of the study sites, data specifications, the registration results, and accuracy assessment are provided.

\subsection{Study Sites and Data Specification}

The study area, located in Maan Mountain forest park (30.52 N, 114.46 E), Wuhan City, Hubei Province, China. Two study sites were selected for data collection as shown in Fig.4. The data specifications of the two sites are listed in Table.2. Due to the limitation of the measurement range $(100 \mathrm{~m})$ of Velodyne Puck VLP-16, flight height of the UAV were set to about $70 \mathrm{~m}$. The flight speed was about $3 \mathrm{~m} / \mathrm{s}$.

\begin{tabular}{ccc}
\hline \hline & Site 1 & Site 2 \\
\hline Flight Height $(\mathrm{m})$ & 70 & 70 \\
Flight Speed $(\mathrm{m} / \mathrm{s})$ & 3 & 3 \\
Number of Points & $3,553,000$ & $2,412,000$ \\
Point Density $\left(\mathrm{pt} / \mathrm{m}^{2}\right)$ & 15 & 15 \\
Number of & 312 & 265 \\
Multispectral image & & \\
\hline \hline
\end{tabular}

Table 2. Data specifications.

\subsection{Results of Multisensory Registration}

The multisensory data is registered using the proposed workflow. Registration results are shown in Fig.5. From a visual inspection, the proposed method achieved an accurate registration between the laser scanning points and the multispectral DOM.
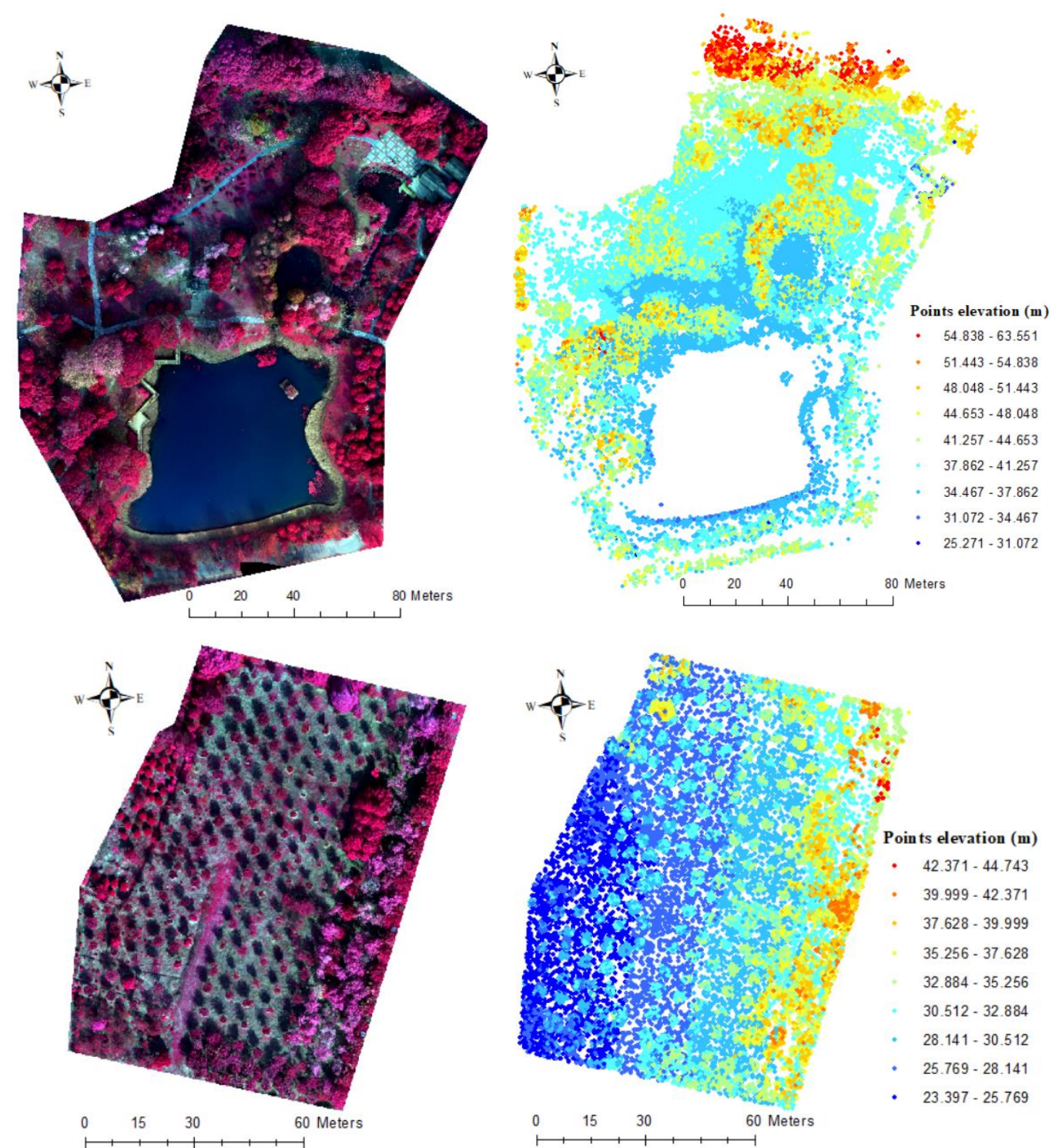

Fig.5 Registration results of the two study sites. 


\subsection{Registration Accuracy Evaluation}

To further evaluate the accuracy of the registration results, corresponding feature points are selected manually from laser scanning point clouds and the multispectral DOM to check the accuracy. The selected feature points should be easily recognized both in image and laser scanning points, and evenly distributed in the study sites. The registration error of feature points selected in the two sites are plotted in Fig.6 (10 points for each test site). The average registration errors of Site 1 and Site 2 are 0.98 and 1.04 pixels, respectively. It demonstrates the high potential of the proposed low-cost system for multisensory data fusion.

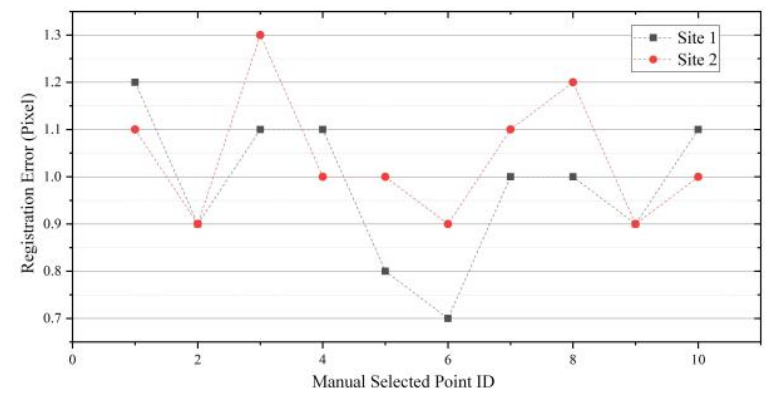

Fig.6 Error distribution of the manual selected points.

\begin{tabular}{ccccc}
\hline \hline & Average & Min & Max & RMSE \\
\hline Site 1 & 0.98 & 0.7 & 1.2 & 0.99 \\
Site 2 & 1.04 & 0.9 & 1.3 & 1.04 \\
\hline \hline
\end{tabular}

\section{CONCLUSION}

In this work, a low-cost multisensory UAV system, named Kylin Cloud, is integrated for forest applications. The Kylin Cloud is composed of a GNSS reveiver, an IMU, a global shutter camera, a multispectral camera, a laser scanner, provides the fusion of the imagery and laser scanning data for reliable forest inventory. Experiments showed that the registration errors of the multispectral DOM and laser scanning data are bellow one pixel, demonstrating a high potential of the proposed low-cost system. 3D individual tree mapping using the registered multisensory data will be explored in the near future.

\section{ACKNOWLEDGEMENTS}

This study was jointly supported by the National Science Fund for Distinguished Young Scholars (No. 41725005), National Natural Science Foundation Project (No. 41531177), and National Key Research and Development Program of China (No. 2016YFF0103501).

\section{REFERENCES}

Abayowa, B.O., Yilmaz, A., Hardie, R.C., 2015. Automatic registration of optical aerial imagery to a LiDAR point cloud for generation of city models. ISPRS Journal of Photogrammetry and Remote Sensing 106, 68-81.

Dai, W., Yang, B., Dong, Z., Shaker, A., 2018. A new method for 3D individual tree extraction using multispectral airborne LiDAR point clouds. ISPRS journal of photogrammetry and remote sensing 144 , 400-411.

García, M., Riaño, D., Chuvieco, E., Salas, J., Danson, F.M.,
2011. Multispectral and LiDAR data fusion for fuel type mapping using Support Vector Machine and decision rules. Remote Sensing of Environment 115, 1369-1379.

Jaakkola, A., Hyyppä, J., Yu, X., Kukko, A., Kaartinen, H., Liang, X., Hyyppä, H., Wang, Y., 2017. Autonomous collection of forest field reference- - The outlook and a first step with UAV laser scanning. Remote Sensing 9, 785.

Li, J., Yang, B., Chen, C., Huang, R., Dong, Z., Xiao, W., 2018. Automatic registration of panoramic image sequence and mobile laser scanning data using semantic features. Isprs Journal of Photogrammetry \& Remote Sensing 136, 41-57.

Li, J., Yang, B., Cong, Y., Cao, L., Fu, X., Dong, Z., 2019. 3D Forest Mapping Using A Low-Cost UAV Laser Scanning System: Investigation and Comparison. Remote Sensing 11, 717.

Puttonen, E., Litkey, P., Hyyppä, J., 2010. Individual tree species classification by illuminated-Shaded area separation. Remote Sensing 2, 19-35.

Tooke, T.R., Coops, N.C., Goodwin, N.R., Voogt, J.A., 2009. Extracting urban vegetation characteristics using spectral mixture analysis and decision tree classifications. Remote Sensing of Environment 113, 398-407.

Wang, Y., Hyyppä, J., Liang, X., Kaartinen, H., Yu, X., Lindberg, E., Holmgren, J., Qin, Y., Mallet, C., Ferraz, A., 2016. International benchmarking of the individual tree detection methods for modeling 3-D canopy structure for silviculture and forest ecology using airborne laser scanning. IEEE Transactions on Geoscience and Remote Sensing 54, 5011-5027.

Yang, B., Dai, W., Dong, Z., Liu, Y., 2016. Automatic forest mapping at individual tree levels from terrestrial laser scanning point clouds with a hierarchical minimum cut method. Remote Sensing 8,372 . 